same age group with fever but without febrile seizure. (Kimia AA, Capraro AJ, Hummel D, Johnston P, Harper MB. Utility of lumbar puncture for first simple febrile seizure among children 6 to 18 months of age. Pediatrics Jan 2009;123:6-12). (Respond: Amir A Kimia MD, Division of Emergency Medicine, Children's Hospital Boston, 300 Longwood Ave, Boston, MA 02115. E-mail: amir.kimia@childrens.harvard.edu).

COMMENT. The AAP practice parameter emphasizes age as the main criterion for considering LP in young patients with first simple febrile seizure (AAP Practice parameter. Pediatrics 1996;97:769-772; discussion 773-775). In contrast, pediatric emergency medicine physicians consider symptoms of meningitis, independent of the seizure and fever, are required before LP is performed (Rosenberg NM et al. Pediatr Emerg Care 1992;8:300301)(Nozicka C. Pediatrics 1997;99:306-307). A representative of the AAP committee responded that practice guidelines should not eliminate clinical judgment and are not mandatory (Duffner PK. Pediatrics 1997;99:306-307).

A recent 2008 report of patients with febrile seizures (FS) treated in a 1-year period, 2005-06, at an East Carolina University-affiliated hospital found that FS were first episodes in $64 \%$, simple in $77 \%$, and complex in $23 \%$. At 100 consecutive FS patient visits, LP was performed in $14 \%$; 11 had complex FS and 3 were simple FS (no SFS patient was aged $<12$ months and only 1 was aged $<18$ months). Of 49 patients with FSFS, only 3 (6\%) underwent LP, and age (13 months) was an indication in 1. In a total of 77 patients with SFS, 3.9\% underwent LP, compared with $48 \%$ of those with complex FS. (Millichap JJ et al. Pediatr Neurol 2008;39:381-386). All were negative for evidence of bacterial or viral meningitis. Age was not a prominent criterion for LP. Complex FS was the main criterion for LP. Other factors significantly more prevalent in patients with LP compared to those without were an abnormal neurological examination, and signs of infection prompting blood culture and empiric antibiotic treatment. In the year 2006, the proportion of patients aged 6-18 months with first SFS who received LP was 3.5\% (1 of 28) in the East Carolina and 10\% (3 of 29) in the Boston studies. The decision to perform LP based on clinical indications and the physician's judgment is supported by the findings and recommendations of both groups.

\title{
EFFECT OF FEBRILE SEIZURES ON OXIDANT STATUS
}

Erythrocyte malondialdehyde (EMA), glutathione peroxidase (GPO), and superoxide dismutase (SDM) levels were assessed in 31 children with a febrile seizure and 30 without, in a study at Dokuz Eylul University, Izmir, Turkey. Febrile seizures were simple in 25 (80\%) and complex in $6(20 \%)$ patients. EMA and GPO levels were significantly higher and SDM significantly lower in the febrile seizure group. Levels were not different in the simple and complex seizure groups. Changes in antioxidant enzyme levels are evidence of oxidative stress that may lead to brain cell damage following febrile seizures. (Gunes S, Dirik E, Yis U et al. Oxidant status in children after febrile seizures. Pediatr Neurol Jan 2009;40:47-49). (Respond: Dr Gunes, Department of Pediatrics, Dokuz Eylul University School of Medicine, 35340 Balcova, Izmir, Turkey. E-mail: sezgin gunes(a,yahoo.com).

COMMENT. The authors conclude that the oxidant-antioxidant balance is disturbed in children with febrile seizures, and changes in lipid peroxidation and level of antioxidant 
enzymes may result in neuronal cell damage after febrile seizures. That febrile seizures may be less benign than generally assumed, as suggested by this study, is also apparent from a recent report of MRI abnormalities that followed both simple and complex febrile seizures (Hesdorffer DC et al. Epilepsia 2008;49:765-771), and an increased risk of sudden unexpected death during 2 years after a first febrile seizure (Vestergaard $\mathrm{M}$ et al, Lancet 2008;372:457-463) (Ped Neur Briefs 2008;22:47-48 and 68-69). Recommendations for the management of febrile seizures are outlined in a report of the Italian League Against Epilepsy (Epilepsia Jan 2009;50(Suppl 1):2-6), with reference to the AAP Guidelines 1996, 1999, and to Fukuyama Y et al. Brain Dev 18:479-484. The possible benefits of prolonged antiepileptic therapy to prevent recurrence of febrile seizures must be weighed against the reported adverse cognitive effects. Currently, chronic prophylactic treatment is usually discouraged, except in some patients with recurrent complex febrile seizures. More effective antipyretic treatment, rapid viral testing and antiviral therapy should provide a more specific approach to the prevention of recurrence of febrile seizures (Millichap JJ et al. J Infect Dis 2008;198:1093-4).

\section{RISK FACTORS FOR NEONATAL SEIZURES}

The association between maternal characteristics and birth admission seizures in a cohort of 2.3 million California children born at >36 weeks' gestation between 1998 and 2002 was evaluated using database statistics at the University of California San Francisco and Davis, CA. The incidence of birth admission seizures was $0.95 / 1000$ live births. The risk of seizures was higher in male infants. Women age 40 years and older who were nulliparous, had diabetes mellitus, intrapartum fever, or infection (chorioamnionitis) or delivered at $>42$ weeks had an increased risk of delivering an infant with seizures. Maternal/race ethnicity was significantly associated with seizures, with a lower risk in infants of Hispanic and Asian compared with Caucasian and African-American mothers. Identifying antenatal and intrapartum risk factors for neonatal seizures might lower infant neurological morbidity and mortality. Late delivery is one risk factor that might be avoided. (Glass HC, Pham TN, Danielsen B, Towner D, Glidden D, Wu YW. Antenatal and intrapartum risk factors for seizures in term newborns: a population-based study, California 1998-2002. J Pediatr Jan 2009;154:24-28). (Reprints: Hannah C Glass, University of California San Francisco, Department of Neurology Box 0663, 521 Parnassus Ave, C-215, San Francisco, CA 94143. E-mail: Hannah.Glass@ucsf.edu.).

COMMENT. Previous reports found a higher incidence of neonatal seizures, from 1 to 3.5 per 1000 in term infants, but other studies included seizures up to 28 days of life. The increased risk of seizures at birth with maternal intrapartum fever and infection remains elevated even when meningitis or neonatal infection is excluded. The risk is higher in febrile multiparous compared to nulliparous women. A higher risk of seizures in infants born by cesarean section during night hours is unexplained.

Unexplained seizures in an infant. A girl born by cesarean section at 38 weeks after placental detachment developed generalized tonic-clonic seizures and tremors at 2 months. Blood tests, EEG and MRI were normal. The mother was receiving amitriptyline for postnatal depression and tension headache. The infant was treated with phenobarbital but 